\title{
COMUNIDAD FITOPLANCTONICA EN LOS RÍOS CURARAY, ARABELA Y NAPO (AMAZONÍA PERUANA)
}

\author{
Rosa ISMIÑO'; Áurea GARCIA'; Homero SÁNCHEZ; Werner CHOTA-MACUYAMA'; Gladys \\ VARGAS $^{1}$; Christian NOLORBE ${ }^{1}$; Salvador TELLO ${ }^{1}$; Carmen GARCÍA-DÁVILA ${ }^{1}$
}

1 Instituto de Investigaciones de la Amazonía Peruana (IIAP). Carretera Iquitos Nauta km 4.5, Iquitos, Perú. Programa para el Uso y Conservación del Agua y sus Recursos - AQUAREC, Apartado 784, Iquitos, Perú. E-mail: rayo14@hotmail.com.

\section{RESUMEN}

Se analizaron la composición, distribución y abundancia de organismos fitoplanctónicos colectados en los periodos de creciente y vaciante del año 2012, en siete estaciones de colecta en la cuenca de los ríos Napo, Arabela y Curaray. En total fueron colectadas 70 muestras de agua (28 muestras para análisis cuantitativo y 42 muestras para análisis cualitativo) que fueron conservadas en formol al 5\%. El análisis cualitativo muestra un total de 72 especies de microalgas, distribuidas en 45 géneros, 25 familias y 7 divisiones. El análisis cuantitativo indica que tanto la riqueza específica como la densidad fitoplanctónica fueron mayores en el periodo de creciente (52 especies, y 22,200 individuos..$^{-1}$ ) que en vaciante (41 especies, y 8,800 individuos. $1^{-}$ ${ }^{1}$ ). En ambos periodos, las Bacillariophytas fueron las algas predominantes entre las divisiones presentes con 12000 individuos. $1^{-1}$. La composición y concentración de fitoplancton en estos cuerpos de agua nos permite concluir que estos ambientes presentan condiciones normales, debido a que aún están presentes los organismos del primer eslabón de la cadena trófica.

PALABRAS CLAVE: Napo, Arabela, Curaray, Peruana, fitoplancton, diversidad, abundancia.

\section{PHYTOPLANKTON COMMUNITY IN CURARAY, ARABELA AND NAPO RIVERS (PERUVIAN AMAZON)}

\section{ABSTRACT}

The goal of this study was to analyze the composition, distribution and abundance of phytoplankton organisms collected from seven sampling stations during the growing and dry seasons of 2012 in the Napo, Arabela and Curaray, rivers. A total of 70 water samples ( 28 for quantitative and for qualitative analysis) were collected and preserved in formalin at $5 \%$. Qualitative analysis showed 72 species of microalgae distributed in 45 genera, 25 families and 7 divisions. Quantitative analysis showed that both, specific richness and phytoplankton density were higher in the growing season ( 52 species , 22,200 ind.1-1) when compared to dry season ( 41 species , 8,800 ind.l-1 ) being the Bacillariophytas the predominant group in both hydrological periods. The phytoplankton composition and concentration recorded in these rivers indicate that these environments have normal conditions for aquatic life since most of the organisms that form the first link of the trophic chain are present.

KEYWORDS: Napo, Arabela, Curaray, phytoplankton, diversity, abundance. 


\section{INTRODUCCIÓN}

Los ecosistemas acuáticos amazónicos constituyen la mayor cuenca hidrográfica del mundo y representan el reservorio de biota acuática más diversa existente en nuestro planeta (Reis et al., 2004; Barthem \& Goulding, 2007; Ortega, 2007). Por su gran extensión geográfica y creciente ocupación demográfica los cuerpos de agua amazónicos se encuentran muy sensibles y amenazados, tanto en la calidad de sus aguas, como en la diversidad y abundancia de los organismos vivos que en ella habitan (Ortega, 2007). El conocimiento de las comunidades fitoplanctónicas en la Amazonía peruana es aún escaso (Aldave, 1989) y la poca información generada está relacionada a cuerpos de agua cercanos a la ciudad de Iquitos (Carey, 1976, 1977; Carey \& Documet 1977; Aldave 1989).

En los sistemas acuáticos, el fitoplancton constituye el primer eslabón en la cadena trófica, ocupando distintos nichos ecológicos y representando alimento para los otros niveles tróficos (Roldán 1992). La abundancia, composición y distribución del fitoplancton en los cuerpos de agua son indicadores de la calidad ambiental de estos ecosistemas, debido a que su presencia representa buenos niveles de oxígeno (el fitoplancton produce oxígeno) y su ausencia puede representar una de las primeras señales de alteraciones provocadas por las actividades antrópicas, debido a su alta sensibilidad a los cambios en las condiciones ambientales y a su corto ciclo de vida (Quiroz, et al., 2004). La abundancia, composición y distribución del fitoplancton pueden ser alteradas también por el régimen hidrológico, mayor determinante de las variaciones de la biota fitoplanctónica de un cuerpo de agua en un mismo punto cada año (Allan, 1995; Reynolds, 1997). Es decir, para tener una real comprensión sobre cuáles son los factores que están alterando la abundancia, composición y distribución del fitoplancton en un determinado cuerpo de agua, es necesario realizar monitoreos en distintos periodos hidrológicos.

Hasta la fecha no existen registros bibliográficos sobre las comunidades fitoplanctónicas de los ríos Arabela, Curaray y Napo; solo hay escasas informaciones provenientes de evaluaciones rápidas de impacto ambiental realizadas en la cuenca del río Napo (Walsh Perú. 2006, GEMA. 2006; ERM, 2007). El presente estudio levanta información sobre los organismos fitoplanctónicos en los ríos Arabela, Curaray y Napo que servirá de línea de base para futuras evaluaciones de la composición, distribución y diversidad de estos organismos en esta área.

\section{METODOLOGÍA}

\section{Área de estudio y colecta de muestras biológicas}

Las muestras fueron colectadas en siete estaciones de muestreo (Figura 1), distribuidas de la siguiente manera: dos en el río Arabela (Panguana = $\mathrm{A} 1$, Flor de $\mathrm{Coco}=\mathrm{A} 2$ ); tres en el río Curaray (Arica $=\mathrm{C} 1$, Urbina $=\mathrm{C} 2$, Shapajal $=\mathrm{C} 3$ ) y dos en la cuenca media del río Napo a 500 metros aguas arriba y 500 metros aguas abajo del delta del río Curaray (Nuevo Defensor $=$ N1, Boca del Curaray $=$ N2).

En cada estación de muestreo se registraron los parámetros físicos y químicos del agua (temperatura, $\mathrm{pH}$, oxígeno, conductividad y sólidos en suspensión), los cuales se midieron en la orilla y en el centro del cuerpo de agua con el auxilio de un analizador multi-parámetros marca YSI, modelo 556 MPS. Además, fue registrada la transparencia del agua con ayuda de un disco de Secchi.

Las colectas fueron realizadas entre abril-mayo y octubre-noviembre del año 2012, comprendiendo los períodos hidrológicos de creciente y vaciante. Fueron colectadas un total de 70 muestras de agua. Para los análisis cuantitativos de cada período hidrológico fueron colectadas dos muestras de agua por estación de muestreo (orilla y centro), sumando 28 muestras en los 2 períodos, según la metodología de Roldan (1992), que consiste en filtrar 100 litros de agua a través de una red cónica de 25 micras de abertura de malla. Para los análisis cualitativos fueron realizados tres arrastres de 15 minutos cada uno por estación de colecta, realizando un total de 42 muestras en creciente y vaciante. Las muestras de agua fueron preservadas en frascos de boca ancha de $100 \mathrm{ml}$ (rotuladas por estación de colecta) conteniendo formol al $5 \%$ hasta su análisis posterior en el laboratorio de cultivos auxiliares del IIAP.

\section{Identificación de organismos fitoplanctónicos}

La búsqueda y visualización de los organismos fitoplanctónicos fueron realizadas con auxilio de un microscopio binocular simple (marca Leica, modelo ICC50 HD) y el conteo de los mismos con una cámara de Neubauer. La identificación de los organismos colectados fue realizada en base a claves de identificación dicotómicas, especializadas para organismos fitoplanctónicos (Aldave, 1989; Bicudo, 1969 y 2006; Bourrelly, 1968,1970 y1972; Fernández, 1982; Acleto, 1998; Cadima, 2014).

\section{ANÁLISIS DE DATOS}

Para cada grupo de organismos analizados, se determinó el número de individuos por especie; especies por estaciones o lugares de muestreo y 
abundancia relativa del total de especímenes (en porcentaje). Se emplearon los índices de diversidad de especies efectivas (Jost, 2006), equidad u homogeneidad de Shannon (J), y dominancia de Simpson (D), con auxilio del programa informático Past para Windows (vs. 2.09).

\section{RESULTADOS Y DISCUSIÓN}

\section{Composición, abundancia y diversidad global del fitoplancton}

Con relación a la composición fitoplanctónica global, en las siete estaciones de muestreo fueron identificadas un total de 72 especies, distribuidas en 45 géneros, 25 familias y 7 divisiones (Tabla 1). El número de especies registradas en época de creciente fue mayor que en la época de vaciante (52 y 42 especies respectivamente). Resultados semejantes fueron reportados en la composición del fitoplancton de ríos y lagos amazónicos de la cuenca del río Putumayo por Núñez - Avellaneda (2005), registrando mayor cantidad de especies en aguas altas (293) y menor cantidad en aguas bajas (206).

En el área evaluada, la mayor abundancia de fitoplancton se encontró en el período de creciente (22200 individuos. $\left.1^{-1}\right)$, a diferencia del periodo de vaciante en que los organismos fitoplanctónicos

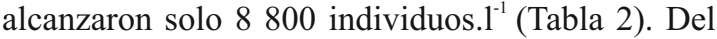
total de organismos registrados la división Bacillariophyta represento el 54\% (12 000 individuos. $\left.1^{-1}\right)$. Estas algas posiblemente son el grupo taxonómico que mejor se adapta a vivir en ambientes lóticos, ya que están presentes durante todo el año y no presentan complicaciones en su ciclo de vida, ecológicamente, además, son más conocidas que otros grupos (Round, 1993). El segundo grupo en abundancia fueron las Chlorophytas con 23\% (5000 individuos..$\left.^{-1}\right)$. Los demás grupos representaron de 4 al $11 \%$ de abundancia. Sin bien, la división Chrysophyta no fue representativa en cuanto a densidad especifica dentro del grupo, fue la más abundante en número de individuos de una misma especie (Rhipidodendron huxleyi con 2400 individuos. $1^{-1}$ ), seguido de las divisiones Bacillariophyta (Eunotia formica $=1700$ individuos..$^{-1}$ ) y Chlorophyta (Hyalotheca dissiliens

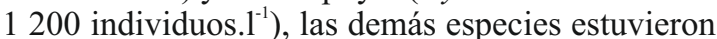
presentes en menor densidad poblacional ( 100 a 900 individuos..$\left.^{-1}\right)$. Otros estudios realizados en la Amazonía peruana mencionan que en ríos de aguas blancas las Bacillariophytas son el grupo fitoplanctónico predominante, seguido de las Chlorophytas, lo cual concuerda con nuestros resultados (Uherkovich, 1976, Uherkovich \& Raid, 1979).
Es importante mencionar que otros grupos fitoplanctonicos de algas como las Cyanophytas y Xantophyta solo fueron observadas en la época de creciente, mientras que el grupo de las Dinophyta estuvo presente únicamente en la época de vaciante. Esta fluctuación temporal de las algas, predominando en algunos períodos y desapareciendo en otros, o haciéndose extremadamente raras, es debido a las condiciones del ambiente acuático en que se encuentran, abandonando el espacio competitivo durante cierto tiempo pasando a una fase latente (Wetzel 2001).

\section{Composición, abundancia y diversidad del fitoplancton por cuencas hidrográficas y estaciones de muestreo}

Globalmente, la cuenca del Curaray fue la que presentó la mayor densidad de organismos fitoplanctónicos (9 300 individuos. $1^{-1}$ ), mientras que la cuenca del río Napo solo registró 5900 individuos. $1^{-1}$ (Figura 2). El Curaray también fue el río que presento la mayor diversidad de especies fitoplanctónicas (62 especies). En tanto que menor cantidad de especies fue registrada en el río Arabela (44 especies).

El mayor porcentaje de organismos fitoplanctonicos en todos estos ríos fue registrado en el periodo de aguas altas. En el río Curaray se registraron 44 especies $(71 \%)$ en creciente, en tanto que en vaciante solo se registraron 18 especies (29\%). Este mismo patrón fue observado en los ríos Napo (33 especies durante el período de aguas altas y 16 en aguas bajas) y Arabela (24 especies en el periodo de creciente y 20 especies en vaciante). Estos resultados concuerdan a los reportados por Núñez-Avellaneda (2005) quien señalo este mismo patrón para el río Putumayo, pero son discordantes con los reportados por Aprile \& Mera (2007), que reportaron mayor diversidad de especies en el periodo de aguas bajas en la cuenca del río Urubuí (Amazonía brasilera). La diversidad de organismos fitoplanctonicos está relacionada con el tipo de agua, la morfología y cantidad de nutrientes que recibe el cuerpo de agua de sus tributarios. En el caso del río Putumayo el autor atribuye estos resultados al incremento de la conductividad, sólidos en suspensión, calcio y los nitratos que provienen del lavado de los suelos y de las lluvias. En tanto que Aprile \& Mera (2007) atribuyen estos resultados al elevado estado de conservación de los bosques que rodean el área evaluada, que garantiza la provisión de nutrientes oriundos de la descomposición e lixiviación de la vegetación que anualmente son redistribuidos en la planicie de inundación por acción del pulso de inundación. La mayor diversidad 
registrada en nuestros resultados en el periodo de aguas altas, puede deberse a que en el área de estudio, la planicie de inundación es bastante más reducida que en la Amazonía brasileña, por lo que existe una menor contribución de material aloctono de las planicies. En el caso del río Arabela esta situación se agudiza más posiblemente porque este río corre por la llanura amazónica, donde existen formaciones antiguas del precámbrico y paleozoico, con rocas predominantemente ácidas, compuestos de cuarzo que aportan bajas cantidades de minerales (Schlesinger ,1996). Por esta razón, si se compara la química de estos ríos con el promedio mundial de agua dulce, se observa que los sistemas amazónicos tienen menos del 5\% del contenido catiónico del promedio a escala mundial y específicamente el calcio está por debajo del $0,5 \%$, cuando mundialmente este catión tiene un promedio de 5,5\%. (Furch, 1984).

Las estaciones de muestreo que presentaron mayor cantidad de microalgas fueron las estaciones A1 (Panguana) con 4800 individuos. $1^{-1}$, seguido de

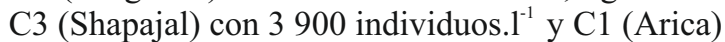

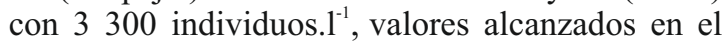
período de creciente. Las estaciones que presentaron menor cantidad de microalgas fueron la estación de

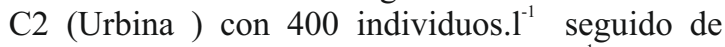
N1(Nuevo defensor) con 900 individuos. $1^{-1}$ en época de vaciante (Tabla 2). Las especies de fitoplancton presentes en el mayor número de estaciones de colecta fueron Rhipidodendron huxleyi (división Chrysophyta), seguida por Eunotia formica, Eunotia convexa y Surirella linearis (división Bacillariophytas), debido a que estuvieron presentes en 7 y 6 estaciones de colecta del área de estudio y en los 2 ciclos hidrológicos. La presencia de las algas de la división Chrysophytas está relacionada con la pobreza de nutrientes en los cuerpos de agua (Gonzales, 1988). La presencia de Oscillatoria tenuis (División Cyanophyta) en cuatro de los siete estaciones evaluadas, es otro indicador de la escasa concentración de nutrientes en el área de estudio, ya que estas algas son capaces de sobrevivir en ambientes pobres en nutrientes, debido a su capacidad de fijar nitrógeno libre que se encuentra en estado gaseoso (Paerl 1988, Reynolds 1997, Peña \& Pinilla, 2002).

\section{Indice de diversidad de fitoplancton}

En el periodo de creciente, las estaciones de Nuevo Defensor y Boca Del Curaray presentaron altos índices de diversidad (14,61 y 14,28 especies efectivas, respectivamente); sin embargo, estas estaciones de muestro presentaron un bajo índice de dominancia y una alta homogeneidad. Por lo tanto, las especies están mejor distribuidas en estos puntos de muestreo. En cambio, la estación Shapajal presentó el valor más bajo en relación al índice de diversidad con 8,21 especies efectivas (Tabla 3). Por ende, Shapajal tiene solamente $56,1 \%$ del total de la diversidad que tiene la estación de Nuevo Defensor. Estas diferencias probablemente se deban a que las estaciones con mayor índice de diversidad se encuentran ubicadas en el río Napo, el cual se caracteriza por presentar aguas blancas ricas en nutrientes. No obstante, el Arabela y Curaray, son ríos de aguas intermedias o mixtas, los cuales tienen una coloración marrón clara, parecidos a los de la cuenca del rio Tigre y sus principales tributarios los ríos Macusari y Corrientes, quienes tienen su origen en el piedemonte amazónico de los andes ecuatorianos. Estos ríos no son productivos, pero son transportadores de nutrientes, por lo cual son importantes dentro del ecosistema amazónico (Maco, 2006)

En vaciante, la estación Panguana y Boca del Curaray presentaron altos índices de diversidad en cuanto a especies efectivas con 10,69 y 8,53 respectivamente. Sin embargo, los valores más bajos se presentaron en Urbina y Shapajal con 4,00 y 4,13 especies efectivas cada una. De acuerdo a estos datos, realizando una comparación entre Panguana y Urbina, esta estación tiene solo el $37,4 \%$ del total de diversidad que Panguana. Por lo tanto, existe una pérdida del $62,58 \%$ de diversidad fitoplanctónica. Posiblemente podría ser debido a acciones antrópicas sobre el ecosistema que crean factores de perturbación y agudizan el desequilibrio del mismo.

De acuerdo a los resultados obtenidos en nuestro estudio, podemos concluir que estos ambientes presentan niveles de diversidad y concentración de fitoplancton normales, de acuerdo a las características físicas y químicas del hábitat. 


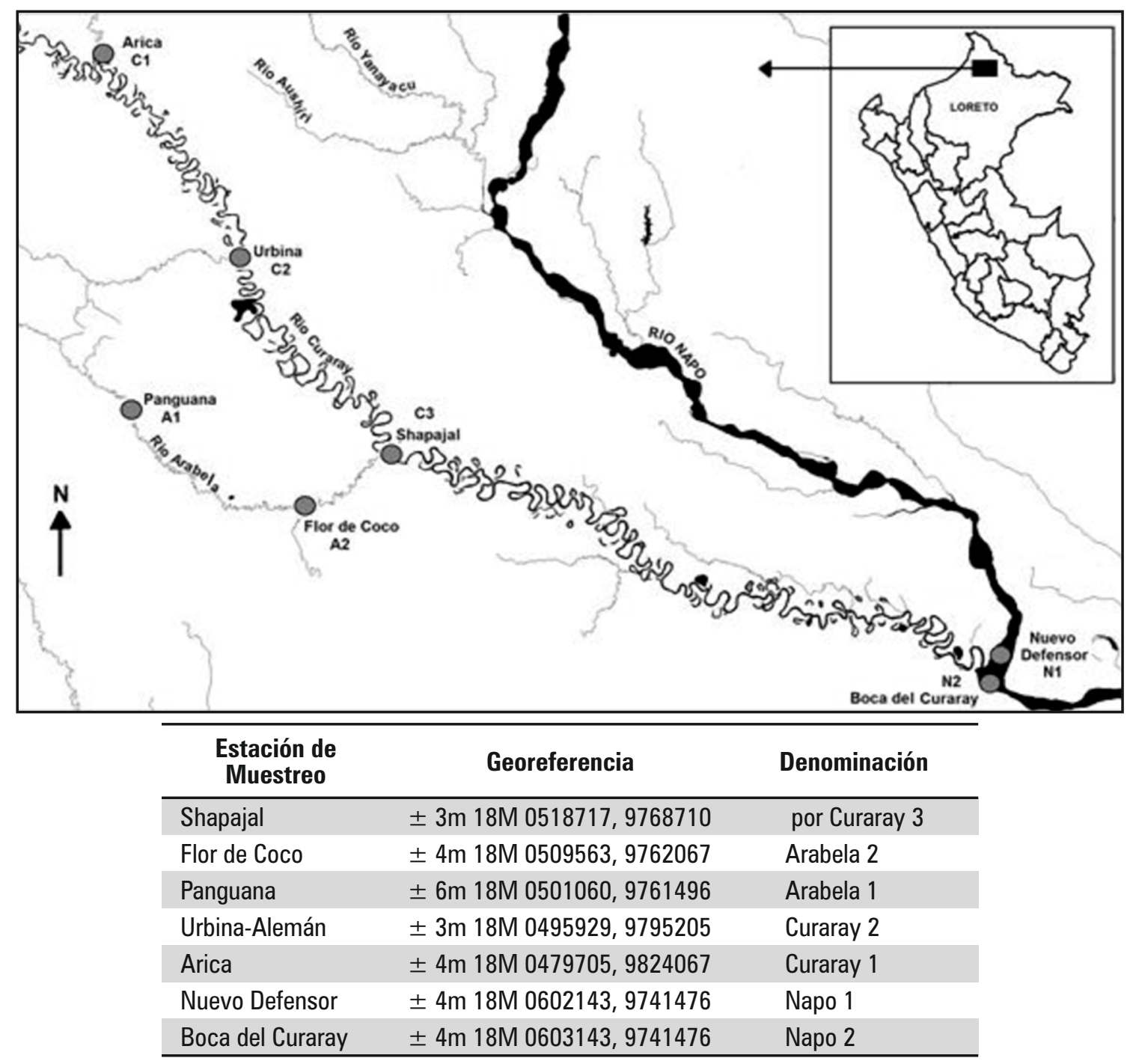

Figura 1. Estaciones de muestreo de las comunidades fitoplanctonicas

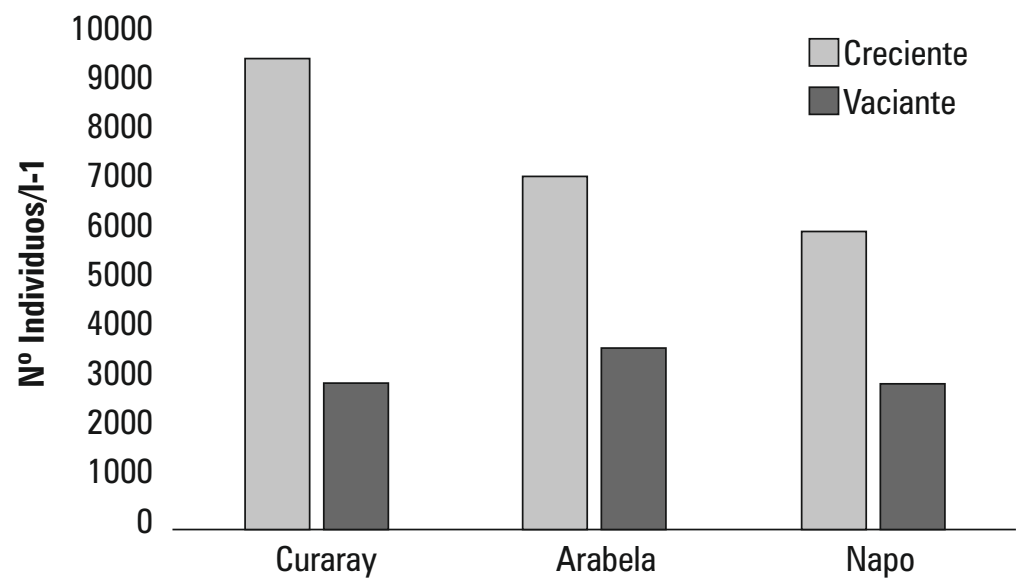

Figura 2.Individuos Fitoplanctónicos por cuenca por periodos hidrológicos 
Tabla 1. Clasificación Taxonómica del Fitoplancton e individuos encontrados en creciente y vaciante.

\begin{tabular}{|c|c|c|c|c|}
\hline DIVISIÓN & FAMILIA & ESPECIE & $\begin{array}{l}\text { Creciente } \\
\text { ind } / /^{1-1}\end{array}$ & $\begin{array}{c}\text { Vaciante } \\
\text { ind } / /^{1-1}\end{array}$ \\
\hline \multirow[t]{3}{*}{ Cyanophyta } & Oscillatoriaceae & Oscillatoria terebriformis & 200 & 0 \\
\hline & & Oscillatoria tenuis. & 1100 & 0 \\
\hline & & Spirulina sp. & 400 & 0 \\
\hline \multirow[t]{8}{*}{ Euglenophyta } & Euglenaceae & Euglena sp. & 200 & 0 \\
\hline & & Trachelomonas volvocina & 200 & 0 \\
\hline & & Trachelomonas armata & 200 & 300 \\
\hline & & TraHcelomonas intermedia & 200 & 0 \\
\hline & & Trachelomonas bacillifera & 100 & 0 \\
\hline & & Strombomonas verrucosa & 0 & 100 \\
\hline & & Strombomonas fluviatilis & 0 & 100 \\
\hline & & Phacus orbicularis & 0 & 100 \\
\hline \multirow[t]{25}{*}{ Bacillariophyta } & Fragilariaceae & Meriodon circulare & 500 & 0 \\
\hline & & Diatoma sp. & 600 & 0 \\
\hline & & Synedra tabulata & 400 & 0 \\
\hline & & Synedra goulardi & 700 & 200 \\
\hline & & Ceratoneis arcus & 800 & 200 \\
\hline & Naviculaceae & Navícula sp. & 100 & 100 \\
\hline & & Navícula tripuntata & 200 & 0 \\
\hline & & Navícula criptocephala & 100 & 200 \\
\hline & & Pinnularia sp. & 1000 & 100 \\
\hline & & Pinnularia nobilis & 500 & 200 \\
\hline & & Pinnularia undulata & 0 & 200 \\
\hline & & Pinnularia viridis & 0 & 400 \\
\hline & & Gyrosigma sp. & 300 & 0 \\
\hline & & Brachisira serians & 0 & 200 \\
\hline & Surirellaceae & Surirella linearis & 1100 & 300 \\
\hline & & Surirella ovalta & 400 & 100 \\
\hline & Eunotiaceae & Eunotia sp. & 600 & 200 \\
\hline & & Eunotia convexa & 900 & 600 \\
\hline & & Eunotia formica & 1700 & 200 \\
\hline & & Eunotia diodon & 700 & 400 \\
\hline & & Eunotia maior & 100 & 200 \\
\hline & & Eunotia robusta & 700 & 300 \\
\hline & & Eunotia veneris & 100 & 0 \\
\hline & & Eunotia faba & 0 & 100 \\
\hline & & Eunotia praerupta & 0 & 200 \\
\hline
\end{tabular}




\begin{tabular}{|c|c|c|c|c|}
\hline DIVISIÓN & FAMILIA & ESPECIE & $\begin{array}{l}\text { Creciente } \\
\text { ind } / /^{1-1}\end{array}$ & $\begin{array}{c}\text { Vaciante } \\
\text { ind } / /^{1-1}\end{array}$ \\
\hline & & Actinella punctata & 200 & 0 \\
\hline & Gomphonemaceae & Gomphonema augur & 100 & 0 \\
\hline & Achnanthaceae & Achnanthes lanceolata & 100 & 0 \\
\hline & Cymbellaceae & Amphora ovalis & 100 & 0 \\
\hline \multirow[t]{25}{*}{ Chlorophyta } & Volvocaceae & Eudorina elegans & 300 & 200 \\
\hline & & Platidorina caudata & 200 & 0 \\
\hline & & Pandorina morum & 100 & 200 \\
\hline & Coccomyxaceae & Elakotothrix lacustris & 200 & 300 \\
\hline & Hydrodictyaceae & Pediastrum duplex & 100 & 0 \\
\hline & Scenedesmaceae & Scenedesmus quadricauda & 800 & 400 \\
\hline & & Scenedesmus ecornis & 200 & 100 \\
\hline & & Scenedesmus acuminatus & 200 & 0 \\
\hline & & Scenedesmus acutus & 0 & 100 \\
\hline & & Sc. Producto capitatus. & 0 & 100 \\
\hline & & Closterium acicularis & 100 & 0 \\
\hline & & Closterium lunula & 500 & 400 \\
\hline & & Closterium malmei & 0 & 200 \\
\hline & Desmidiaceae & Hyalotheca dissiliensis & 1200 & 0 \\
\hline & & Actinastrum sp. & 100 & 0 \\
\hline & & Pleurotaenium sp. & 200 & 0 \\
\hline & & Cosmarium sp. & 0 & 200 \\
\hline & Coelastraceae & Coelastrum reticulatum & 100 & 0 \\
\hline & Oocystaceae & Treubaria sp & 100 & 0 \\
\hline & & Kirchneriella lunatus & 0 & 200 \\
\hline & & Polyedropsis sp. & 0 & 100 \\
\hline & & Tetraedron trigonum & 0 & 300 \\
\hline & Mesotaeniaceae & Gonatozygon kinahani & 400 & 0 \\
\hline & & Gonatozygon aculeatum & 0 & 100 \\
\hline & Zygnemataceae & Spirogyra sp. & 200 & 0 \\
\hline \multirow[t]{5}{*}{ Chrysophyta } & Derepixidaceae & Rhipidodendron huxleyi & 2400 & 500 \\
\hline & Naegeliellaceae & Naegeliella $s p$ & 100 & 0 \\
\hline & Chrysophyceae & Chrysococcus sp & 0 & 100 \\
\hline & Chrysocapsaceae & Chrysopora fenestrata & 0 & 100 \\
\hline & Synuraceae & Synura spinosa & 0 & 100 \\
\hline Xanthophyta & Centritractaceae & Polyedriella sp. & 100 & 0 \\
\hline \multirow[t]{2}{*}{ Dinophyta } & Prorocentraceae & Prorocentrum micans & 0 & 100 \\
\hline & & TOTAL & 22200 & 8800 \\
\hline
\end{tabular}


Tabla 2. Número de individuos fitoplanctónicos por estación de muestreo en el periodo de creciente y vaciante.

\begin{tabular}{lcc}
\hline Estaciones & $\begin{array}{c}\text { Creciente } \\
\text { ind. }^{-1}\end{array}$ & $\begin{array}{c}\text { Vaciante } \\
\text { ind. } .^{-1}\end{array}$ \\
\hline Panguana & 4,800 & 2,400 \\
\hline Flor de coco & 2,200 & 1,200 \\
N. Defensor & 4,100 & 900 \\
\hline Boca Curaray & 1,800 & 1,400 \\
Arica & 3,300 & 1,500 \\
\hline Urbina & 2,100 & 400 \\
\hline Shapajal & 3,900 & 1,000 \\
\hline Total & 22,200 & 8,800 \\
\hline
\end{tabular}

Tabla 3. Índices de diversidad del fitoplancton en el área de estudio en época de creciente y vaciante.

\begin{tabular}{ccccccccccc}
\hline \multirow{2}{*}{ Estaciones } & \multicolumn{4}{c}{ Creciente } & \multicolumn{5}{c}{ Vaciante } \\
\cline { 2 - 12 } & E & A & D & H & E.E & E & A & D & H & E.E \\
\hline A1 & 13 & 48 & 0,11 & 0,91 & 10,26 & 12 & 24 & 2,37 & 0,95 & 10,69 \\
A2 & 11 & 22 & 0,12 & 0,93 & 9,37 & 8 & 12 & 1,9 & 0,92 & 6,73 \\
N1 & 18 & 41 & 0,08 & 0,93 & 14,61 & 7 & 9 & 1,83 & 0,94 & 6,23 \\
N2 & 15 & 18 & 0,07 & 0,98 & 14,28 & 9 & 14 & 2,14 & 0,98 & 8,53 \\
C1 & 18 & 33 & 0,14 & 0,85 & 11,83 & 9 & 15 & 2,12 & 0,96 & 8,32 \\
C2 & 13 & 21 & 0,16 & 0,87 & 9,38 & 4 & 4 & 1,39 & 1 & 4,00 \\
C3 & 13 & 39 & 0,17 & 0,82 & 8,21 & 5 & 10 & 1,41 & 0,88 & 4,13 \\
\hline
\end{tabular}

$\mathrm{E}=\mathrm{N}^{\circ}$ de Especies, $\mathrm{A}=$ Abundancia $\times 100 \mathrm{I}, \mathrm{D}=$ Dominancia, $\mathrm{H}=$ Homogeneidad, $\mathrm{E} . \mathrm{E}=$ Especies efectivas

\section{AGRADECIMIENTO}

Los autores agradecen a la empresa PERENCO PERU PETROLEUM LIMITED por el financiamiento del presente estudio, a través del proyecto "Evaluación hidrobiológica de los ríos Arabela y Curaray (Cuenca del rio Napo)".

\section{REFERENCIAS BIBLIOGRÁFICAS}

Acleto, C.; Zuñiga, R.1998. Introducción a las algas. Perú. Editorial Escuela Nueva S. A. Lima - Perú $.383 \mathrm{pp}$.

Aldave, A.1989. Algas. Editorial Libertad. Trujillo. Perú. 269 pp.

Allan, D. 1995 Stream Ecology, structure and function of running waters, Chapman and Hall, 2-6 Boundary Row, London. 388 pp.
Aprile, F.M. \& Mera, P.A.S. 2007. Fitoplancton e fitoperifiton de um rio de águas pretas da Amazônia periférica do norte, Brasil.Brazilian Journal of Aquatic Science and Technology, 11 (2): 1-14.

Barthem, R. B.; Goulding, M. 2007. Un ecosistema inesperado: A Amazonia revelada pela Pesca. Grafica Biblos, Lima-Perú, 241pp.

Bicudo, C; Bicudo, T. 1969. Algas de aguas continentais brasileras. Fundacao Brasileira para o Desnvolvimento do ensino de ciências. Sao Paulo. Brasil.277 pp.

Bicudo, C; Menezes,M. 2006.Géneros de Algas Continentais Do Brasil.Editora RiMa.489pp.

Bourrely, P. 1972. Les Algues Deau Douce Tomo I les Algues vertes. Editions N. Boubee \& Cie. Paris. 470pp. 
Bourrely, P. 1968. Les Algues Deau Douce Tomo II les Algues jaunes et brunes Chrysophycees, Pheophycees, xanthophycees et Diatomees. Editions N. Boubee \& Cie. Paris. 438pp.

Bourrely, P. 1970. Les Algues Deau Douce Tomo III les Algues bleues et rouges les Eugleniens, Peridiniens et Crytomonadines. Editions N. Boubee \& Cie. Paris. 450pp.

Cadima, M.F y Bicudo, C. 2014, Guia ilustrada de algas de Bolivia división Euglenophyta Universidad Mayor de San Simon .Segunda Edición. 263pp.

Carey, S.P.1976, Desmidias del Lago Moronacocha. Conocimiento UNAP, 1 (2): 27-37.

Carey, S.P. y Documet. M. T. 1977, Tetrasporales y Chlorococcales del plancton superficial del Lago Quistococha, Iquitos, Desmidias del Lago Moronacocha. Conocimiento UNAP, 1 (3): 1321.

ERM.2007.Estudio de impacto ambiental y social de la prospección sísmica 2D de $445 \mathrm{Km}$ en el lote 104. Burlington Resorces - ConocoPhillips. Resumen ejecutivo. 32pp.

Fernández H.A.1982.Guia para el estudio de las algas. Trujillo, Peru.263pp.

Furch, K. 1984. Seasonal variation of the major content of the varzea-lake lago Cameleao , Amazon, Brasil in 1981 and 1982 . Verhandlungen des Internationalen Verein Limnologie, 22: 1288-1293.

GEMA. 2006. EIA de doce pozos petroleros exploratorios- lote 39 .

Gonzáles, A. 1988. El plancton de las aguas continentales. OEA.Venezuela. 130pp.

Jost, L. 2006. Entropy and Diversity. Oikos, 113 (2): 363-375.

Núñez, A.M. 2005. Fitoplancton en Ecosistemas acuáticas de la Cuenca del Río Putumayo (Amazonia Colombo Peruana). Tesis para Optar el grado de Magister en Estudios Amazónicos. Universidad Nacional de Colombia, sede Leticia.94pp.

Maco, G.J. 2006.Tipos de ambientes acuáticos en la Amazonia Peruana. Folia Amazónica, 15 (1-2): 131-140.

Ortega, H. Rengifo, B. Samanez, I., y Palma, C. 2007.Diversidad y el estado de conservación de cuerpos de agua amazónicas en el nororiente del
Perú. Revista Peruana de Biología, 13 (3): 2-4.

Paerl, H. 1988. Growth and reproductive strategies of freshwater blue-green algae (Cyanobacteria).En: Sandgren CD (ed), Growth and reproductive strategies of freshwater phytoplankton, 261-315. Cambridge University Press, Cambridge.

Peña, V.; Pinilla, G.2002. Composición, distribución y abundancia de la comunidad fitoplanctónica de la ensenada de Utria, Pacifico Colombiano. Revista de Biología Marina y Oceanografía, 37 (1): 67-81.

Quiroz, C.H., Solís, Mora, Z. M.L., Molina, A.I y García, R.J.2004. Variación de los organismos fitoplanctonicos y la calidad del agua en el lago de Chapala Jalisco México. Acta Universitaria, $14(1): 1-25$.

Reis, R. E.; Kullander, S. O.; Ferraris, C. J. 2004. Checklist of Freshwater Fishes of South and Central America. Porto Alegre: Edipucrs.

Reynolds, C. S. 1997. Vegetation processes in the pelagica: model for ecosystem theory. Ecology Institute, Germany. 371p.

Roldán, G. 1992. Fundamentos de Limnologia neotropical. Ed. Universidad de Antioquia. Medellin, Colombia.

Round, F.E., Crawford, R.M \& Mann, D.G.1993. The diatoms.Biology \& Morfology of the genera. Cambridge University Press, 747 pp.

Schlesinger, W. 1996. Biogeochemistry. An analysis of global change. Second edition. Academic Press limited.

Uherkovich, G.1976.Algen aus den flussen Rio negro und Rio Tapajós. Amazoniana, 5: 465 515.

Uherkovich,G. y Raí,H.1979. Algen aus dem Rio Negro und seinen Nebenflussen. Amazoniana, 6 (4): 611-638.

Walsh Peru S.A.Estudio de Impacto Ambiental y social de la prospección sísmica 3D. perforación exploratoria del 101. Preparado para Talisman Energy, sucursal peruana.366pp.

Wetzel,R., 2001,Limnology. Saunders CllegePu. $767 \mathrm{pp}$.

Recibido: 16/05/2014

Aceptado para publicación: 07/07/2014 
